\title{
A Generalised-Mutual-Information-Based Oracle for Hierarchical Radiosity
}

\author{
Jaume Rigau, Miquel Feixas, and Mateu Sbert \\ Institut d'Informàtica i Aplicacions \\ Campus Montilivi P-IV, 17071-Girona, Spain \\ jaume.rigau|miquel.feixas|mateu@ima.udg.edu
}

\begin{abstract}
One of the main problems in the radiosity method is how to discretise a scene into mesh elements that allow us to accurately represent illumination. In this paper we present a new refinement criterion for hierarchical radiosity based on the continuous and discrete generalised mutual information measures between two patches or elements. These measures, derived from the generalised entropy of Harvda-Charvát-Tsallis, express the information transfer within a scene. The results obtained improve on the ones based on kernel smoothness and Shannon mutual information.
\end{abstract}

\section{Introduction}

The radiosity method solves the problem of illumination in an environment with diffuse surfaces by using a finite element approach [1. The scene discretisation has to represent the illumination accurately by trying to avoid unnecessary subdivisions that would increase the computation time. A good meshing strategy will balance the requirements of accuracy and computational cost.

In the hierarchical radiosity algorithms [2] the mesh is generated adaptively: when the constant radiosity assumption on a patch is not valid for the radiosity received from another patch, the refinement algorithm will subdivide it in a set of subpatches or elements. A refinement criterion, called oracle, informs us if a subdivision of the surfaces is needed, bearing in mind that the cost of the oracle should remain acceptable. In [34, the difficulty in obtaining a precise solution for the scene radiosity has been related to the degree of dependence between all the elements of the adaptive mesh. This dependence has been quantified by the mutual information, which is a measure of the information transfer in a scene.

In this paper, a new oracle based on the generalised mutual information [5], derived from the generalised entropy of Harvda-Charvát-Tsallis [6], is introduced. This oracle is obtained from the difference between the continuous and discrete generalised mutual information between two elements of the adaptive mesh and expresses the loss of information transfer between two patches due to the discretisation. The results obtained show that this oracle improves on the kernel smoothness-based [7] and the mutual information-based [89] ones, confirming the usefulness of the information-theoretic approach in dealing with the radiosity problem. 


\section{Preliminaries}

\section{$2.1 \quad$ Radiosity}

The radiosity method uses a finite element approach, discretising the diffuse environment into patches and considering the radiosities, emissivities and reflectances constant over them. With these assumptions, the discrete radiosity equation [1] is given by

$$
B_{i}=E_{i}+\rho_{i} \sum_{j \in S} F_{i j} B_{j},
$$

where $S$ is the set of patches of the scene, $B_{i}, E_{i}$, and $\rho_{i}$, are respectively the radiosity, emissivity, and reflectance of patch $i, B_{j}$ is the radiosity of patch $j$, and $F_{i j}$ is the patch-to-patch form factor, defined by

$$
F_{i j}=\frac{1}{A_{i}} \int_{S_{i}} \int_{S_{j}} F(x, y) \mathrm{d} A_{y} \mathrm{~d} A_{x},
$$

where $A_{i}$ is the area of patch $i, S_{i}$ and $S_{j}$ are, respectively, the surfaces of patches $i$ and $j, F(x, y)$ is the point-to-point form factor between $x \in S_{i}$ and $y \in S_{j}$, and $\mathrm{d} A_{x}$ and $\mathrm{d} A_{y}$ are, respectively, the differential areas at points $x$ and $y$. Using Monte Carlo computation with area-to-area sampling, $F_{i j}$ can be calculated:

$$
F_{i j} \approx A_{j} \frac{1}{\left|\mathcal{S}_{i \times j}\right|} \sum_{(x, y) \in \mathcal{S}_{i \times j}} F(x, y),
$$

where the computation accuracy depends on the number of random segments between $i$ and $j\left(\left|\mathcal{S}_{i \times j}\right|\right)$.

To solve the system (11), a hierarchical refinement algorithm is used. The efficiency of this algorithm depends on the election of a good refinement criterion. Many refinement oracles have been proposed in the literature (see [10] for details). For comparison purposes, we review here the oracle based on kernel smoothness (KS), proposed by Gortler et al. [7] in order to drive hierarchical refinement with higher-order approximations. When applied to constant approximations, this refinement criterion is given by

$$
\rho_{i} \max \left\{F_{i j}^{\max }-F_{i j}^{\mathrm{avg}}, F_{i j}^{\mathrm{avg}}-F_{i j}^{\min }\right\} A_{j} B_{j}<\epsilon,
$$

where $F_{i j}^{\max }=\max \left\{F(x, y) \mid x \in S_{i}, y \in S_{j}\right\}$ and $F_{i j}^{\min }=\min \{F(x, y) \mid x \in$ $\left.S_{i}, y \in S_{j}\right\}$ are, respectively, the maximum and minimum radiosity kernel values estimated by taking the maximum and minimum value computed between pairs of random points on both elements, and $F_{i j}^{\mathrm{avg}}=F_{i j} / A_{j}$ is the average radiosity kernel value.

\subsection{HCT Entropy and Generalised Mutual Information}

In 1967, Harvda and Charvát [6] introduced a new generalised definition of entropy. In 1988, Tsallis [11] used this entropy in order to generalise the BoltzmannGibbs entropy in statistical mechanics. 
Definition 1. The Harvda-Charvát-Tsallis entropy (HCT entropy) of a discrete random variable $X$, with $|X|=n$ and $p_{X}$ as its probability distribution, is defined by

$$
H_{\alpha}(X)=k \frac{1-\sum_{i=1}^{n} p_{i}^{\alpha}}{\alpha-1},
$$

where $k$ is a positive constant (by default $k=1$ ) and $\alpha \in \mathbb{R} \backslash\{1\}$ is called entropic index.

This entropy recovers the Shannon discrete entropy when $\alpha \rightarrow 1, H_{1}(X) \equiv$ $-\sum_{i=1}^{n} p_{i} \ln p_{i}$, and fulfils good properties such as non-negativity and concavity.

On the other hand, Taneja [5] and Tsallis [12] introduced the generalised mutual information.

Definition 2. The generalised mutual information between two discrete random variables $(X, Y)$ is defined by

$$
I_{\alpha}(X, Y)=\frac{1}{1-\alpha}\left(1-\sum_{i=1}^{n} \sum_{j=1}^{m} \frac{p_{i j}^{\alpha}}{p_{i}^{\alpha-1} q_{j}^{\alpha-1}}\right)
$$

where $|X|=n,|Y|=m, p_{X}$ and $q_{Y}$ are the marginal probability distributions, and $p_{X Y}$ is the joint probability distribution between $X$ and $Y$.

The transition of $I_{\alpha}(X, Y)$ to the continuous generalised mutual information is straightforward. Using entropies, an alternative form is given by

$$
I_{\alpha}(X, Y)=H_{\alpha}(X)+H_{\alpha}(Y)-(1-\alpha) H_{\alpha}(X) H_{\alpha}(Y)-H_{\alpha}(X, Y) .
$$

Shannon mutual information (MI) is obtained when $\alpha \rightarrow 1$. Some alternative ways for the generalised mutual information can be seen in [13].

\section{Generalised Mutual Information-Based Oracle}

We will see below how the generalised mutual information can be used to build a refinement oracle within a hierarchical radiosity algorithm. Our strategy will be based on the estimate of the discretisation error from the difference between the continuous and discrete generalised mutual information (6) between two elements of the adaptive mesh. The discretisation error based on Shannon mutual information was introduced by Feixas et al. 8] and applied to hierarchical radiosity with good results.

In the context of a discrete scene information channel 4, the marginal probabilities are given by $p_{X}=q_{Y}=\left\{a_{i}\right\}$ (i.e., the distribution of the relative area of patches: $\frac{A_{i}}{A_{\mathrm{T}}}$, where $A_{\mathrm{T}}$ is the total area of scene) and the joint probability is given by $p_{X Y}=\left\{a_{i} F_{i j}\right\}$. Then,

Definition 3. The discrete generalised mutual information of a scene is given by

$$
I_{\alpha}=\frac{1}{1-\alpha}\left(1-\sum_{i \in S} \sum_{j \in S} \frac{a_{i}^{\alpha} F_{i j}^{\alpha}}{a_{i}^{\alpha-1} a_{j}^{\alpha-1}}\right)=\sum_{i \in S} \sum_{j \in S} \tau_{\alpha}\left(a_{i} F_{i j}, a_{i} a_{j}\right),
$$


where, using $1=\sum_{i \in S} \sum_{j \in S} a_{i} a_{j}$ and $\tau_{\alpha}(p, q)=\frac{1}{1-\alpha} \frac{q^{\alpha}-p^{\alpha}}{q^{\alpha-1}}$, the last equality is obtained.

This measure quantifies the discrete information transfer in a discretised scene. The term $\tau_{\alpha}\left(a_{i} F_{i j}, a_{i} a_{j}\right)$ can be considered as an element of the generalised mutual information matrix $I_{\alpha}$, representing the information transfer between patches $i$ and $j$.

To compute $I_{\alpha}$, the Monte Carlo area-to-area sampling (3) is used, obtaining for each pair of elements

$$
\begin{aligned}
I_{\alpha_{i j}} & =\tau_{\alpha}\left(a_{i} F_{i j}, a_{i} a_{j}\right)=\frac{1}{1-\alpha} \frac{a_{i}^{\alpha} a_{j}^{\alpha}-a_{i}^{\alpha} F_{i j}^{\alpha}}{a_{i}^{\alpha-1} a_{j}^{\alpha-1}} \\
& \approx \frac{1}{1-\alpha} \frac{A_{i}}{A_{\mathrm{T}}} \frac{A_{j}}{A_{\mathrm{T}}}\left(1-A_{\mathrm{T}}^{\alpha}\left(\frac{1}{\left|\mathcal{S}_{i \times j}\right|} \sum_{(x, y) \in \mathcal{S}_{i \times j}} F(x, y)\right)^{\alpha}\right) .
\end{aligned}
$$

The information transfer between two patches can be obtained more accurately using the continuous generalised mutual information between them. From the discrete form (8) and using the pdfs $p(x)=q(y)=\frac{1}{A_{\mathrm{T}}}$ and $p(x, y)=$ $\frac{1}{A_{\mathrm{T}}} F(x, y)$, we define

Definition 4. The continuous generalised mutual information of a scene is given by

$$
I_{\alpha}^{\mathrm{c}}=\int_{S} \int_{S} \tau_{\alpha}\left(\frac{1}{A_{\mathrm{T}}} F(x, y), \frac{1}{A_{\mathrm{T}}^{2}}\right) \mathrm{d} A_{y} \mathrm{~d} A_{x} .
$$

This represents the continuous information transfer in a scene. We can split the integration domain and for two surface elements $i$ and $j$ we have

$$
I_{\alpha_{i j}}^{\mathrm{c}}=\int_{S_{i}} \int_{S_{j}} \tau_{\alpha}\left(\frac{1}{A_{\mathrm{T}}} F(x, y), \frac{1}{A_{\mathrm{T}}^{2}}\right) \mathrm{d} A_{y} \mathrm{~d} A_{x}
$$

that, analogously to the discrete case, expresses the information transfer between two patches.

Both continuous expressions, (10) and (11), can be solved by Monte Carlo integration. Taking again area-to-area sampling (i.e., pdf $\frac{1}{A_{i} A_{j}}$ ), the last expression (11) can be approximated by

$$
\begin{aligned}
I_{\alpha_{i j}}^{\mathrm{c}} & \approx A_{i} A_{j} \frac{1}{\left|\mathcal{S}_{i \times j}\right|} \sum_{(x, y) \in \mathcal{S}_{i \times j}} \tau_{\alpha}\left(\frac{1}{A_{\mathrm{T}}} F(x, y), \frac{1}{A_{\mathrm{T}}^{2}}\right) \\
& =\frac{1}{1-\alpha} \frac{A_{i}}{A_{\mathrm{T}}} \frac{A_{j}}{A_{\mathrm{T}}}\left(1-A_{\mathrm{T}}^{\alpha}\left(\frac{1}{\left|\mathcal{S}_{i \times j}\right|} \sum_{(x, y) \in \mathcal{S}_{i \times j}} F(x, y)^{\alpha}\right)\right) .
\end{aligned}
$$

Now, we define 
Definition 5. The generalised discretisation error of a scene is given by

$$
\Delta_{\alpha}=I_{\alpha}^{\mathrm{c}}-I_{\alpha}=\sum_{i \in S} \sum_{j \in S} \Delta_{\alpha_{i j}}
$$

where $\Delta_{\alpha_{i j}}=I_{\alpha_{i j}}^{\mathrm{c}}-I_{\alpha_{i j}}$.

While $\Delta_{\alpha}$ expresses the loss of information transfer in a scene due to the discretisation, the term $\Delta_{\alpha_{i j}}$ gives us this loss between two elements $i$ and $j$. This difference is interpreted as the benefit to be gained by refining and can be used as the base of the new oracle.

From (13), using (9) and (12), we obtain

$$
\Delta_{\alpha_{i j}} \approx A_{i} A_{j} A_{\mathrm{T}}^{\alpha-2} \frac{1}{1-\alpha} \delta_{\alpha_{i j}}
$$

where

$$
\delta_{\alpha_{i j}}=\left(\frac{1}{\left|\mathcal{S}_{i \times j}\right|} \sum_{(x, y) \in \mathcal{S}_{i \times j}} F(x, y)\right)^{\alpha}-\frac{1}{\left|\mathcal{S}_{i \times j}\right|} \sum_{(x, y) \in \mathcal{S}_{i \times j}} F(x, y)^{\alpha} .
$$

Accordingly to the radiosity equation (11) and in analogy to classic oracles, like KS, we consider the oracle structure $\rho_{i} \sigma B_{j}<\epsilon$, where $\sigma$ is the geometric kernel [14. Now, we propose to take the generalised discretisation error between two patches as the kernel $\left(\sigma=\Delta_{\alpha_{i j}}\right)$ for the new oracle based on generalised mutual information $\left(\mathrm{GMI}_{\alpha}\right)$. To simplify the expression of this oracle, we multiply the inequality by the scene constant ${A_{\mathrm{T}}}^{2-\alpha}(1-\alpha)$.

Definition 6. The hierarchical radiosity oracle based on the generalised mutual information is defined by

$$
\rho_{i} A_{i} A_{j} \delta_{\alpha_{i j}} B_{j}<\epsilon
$$

\section{Results}

In this section, the $\mathrm{GMI}_{\alpha}$ oracle is compared with the $\mathrm{KS}$ and $\mathrm{MI}$ ones. Other comparisons, with a more extended analysis, can be found in 14. All oracles have been implemented on top of the hierarchical Monte Carlo radiosity method.

In Fig. 1 we show the results obtained for the $\mathrm{KS}(a)$ and $\mathrm{GMI}_{\alpha}$ oracles with their Gouraud shaded solutions and meshes. In the $\mathrm{GMI}_{\alpha}$ case, we show the results obtained with the entropic indexes 1 (b) (i.e., note that $\mathrm{GMI}_{1}=\mathrm{MI}$ ) and $0.5(c)$. For the sake of comparison, adaptive meshes of identical size have been generated with the same cost for the power distribution: around 19,000 patches and 2,684,000 rays, respectively. To estimate the form factor, the number of random lines has been fixed to 10 .

In Table 1, we show the Root Mean Square Error (RMSE) and Peak Signal Noise Ratio (PSNR) measures for $\mathrm{KS}$ and $\mathrm{GMI}_{\alpha}$ (for 5 different entropic indexes) oracles for the test scene. These measures have been computed with respect to the corresponding converged image, obtained with a path-tracing algorithm 


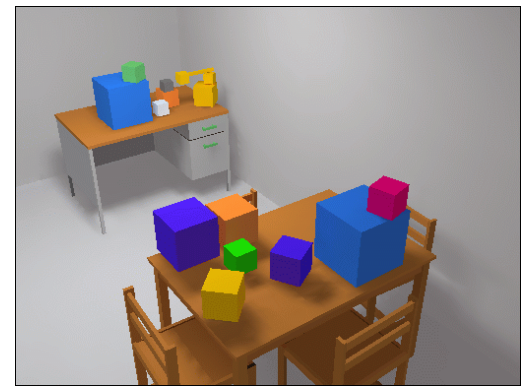

(a.i) $\mathrm{KS}$

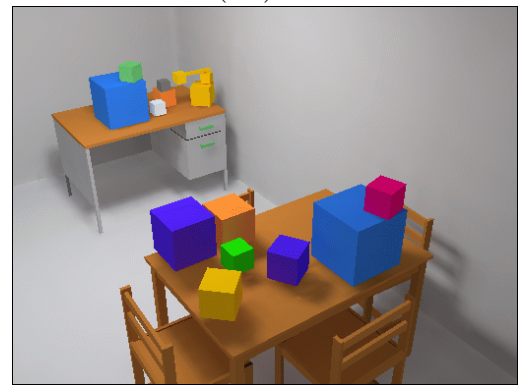

(b.i) $\mathrm{GMI}_{1.00}$

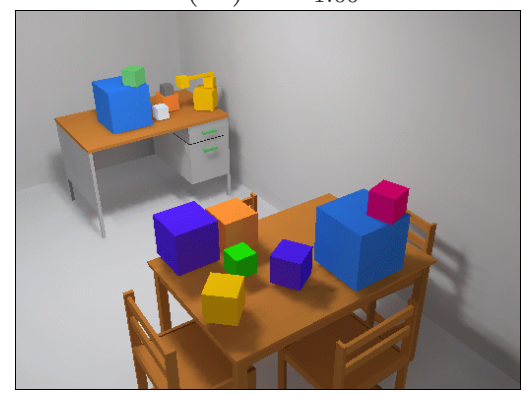

(c.i) $\mathrm{GMI}_{0.50}$

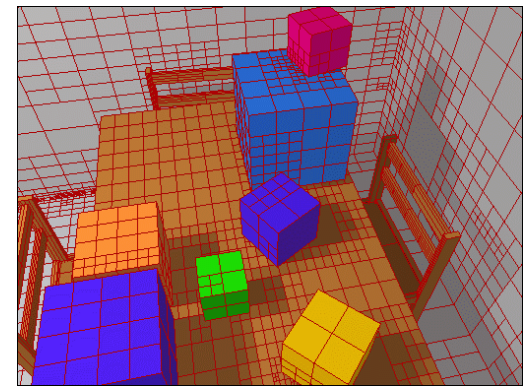

(a.ii) $\mathrm{KS}$

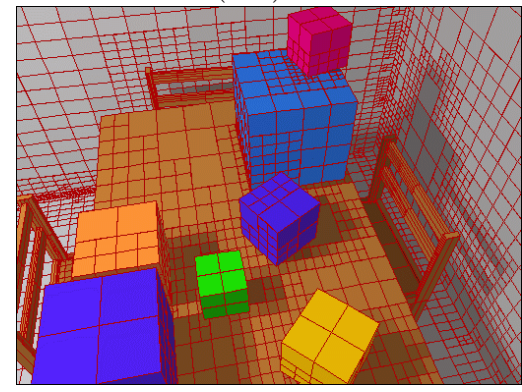

(b.ii) $\mathrm{GMI}_{1.00}$

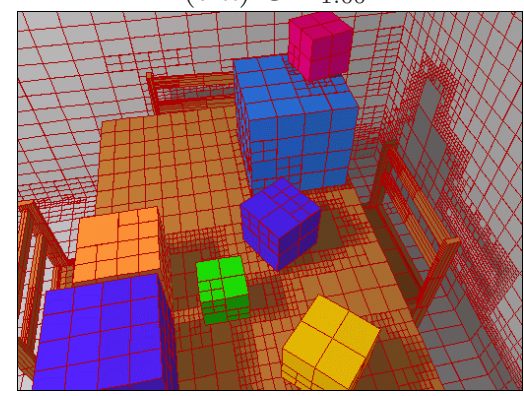

(c.ii) $\mathrm{GMI}_{0.50}$

Fig. 1. (a) KS and $\mathrm{GMI}_{\alpha}$ (entropic indexes (b) 1 and (c) 0.5) oracles. By columns, $(i)$ Gouraud shaded solution of view 1 and $(i i)$ mesh of view 2 are shown.

with 1,024 samples per pixel in a stratified way. For each measure, we consider a uniform weight for every colour channel $\left(\mathrm{RMSE}_{\mathrm{a}}\right.$ and $\left.\mathrm{PSNR}_{\mathrm{a}}\right)$ and a perceptual one $\left(\mathrm{RMSE}_{\mathrm{p}}\right.$ and $\left.\mathrm{PSNR}_{\mathrm{p}}\right)$ in accordance with the sRGB system.

Observe in the view ${ }_{1}$, obtained with $\mathrm{GMI}_{\alpha}$ (Fig. 1 $\left.i . b-c\right)$, the finer details of the shadow cast on the wall by the chair on the right-hand side and also the better-defined shadow on the chair on the left-hand side and the one cast by the desk. In view 2 (Fig. 1 $i i$ ) we can also see how our oracle outperforms the KS, especially in the much more defined shadow of the chair on the right. Note the superior quality mesh created by our oracle. 
Table 1. The RMSE and PSNR measures of the $\mathrm{KS}$ and $\mathrm{GMI}_{\alpha}$ oracles applied to the test scene of Fig. 1 where the $\mathrm{KS}$ and $\mathrm{GMI}_{\alpha \in\{0.5,1\}}$ results are shown. The oracles have been evaluated with 10 random lines between each two elements.

\begin{tabular}{|c|c|c|c|c|c|c|c|c|}
\hline \multirow{2}{*}{ oracle } & \multicolumn{4}{|c|}{ view $_{1}$} & \multicolumn{4}{|c|}{ view $_{2}$} \\
\hline & $\mathrm{RMSE}_{\mathrm{a}}$ & $\mathrm{RMSE}_{\mathrm{p}}$ & |lPSNR & $\mathrm{PSNR}_{\mathrm{p}}$ & $\mathrm{RMSE}_{\mathrm{a}}$ & $\mathrm{RMSE}_{\mathrm{p}}$ & $\mathrm{PSNR}_{\mathrm{a}}$ & $\mathrm{PSNR}_{\mathrm{p}}$ \\
\hline$\overline{\mathrm{KS}}$ & 13.791 & 13.128 & 25.339 & 25.767 & 15.167 & 14.354 & 24.513 & 24.991 \\
\hline$\overline{\mathrm{GMI}_{1.50}}$ & 11.889 & 11.280 & 26.628 & 27.084 & 13.046 & 12.473 & 25.821 & 26.211 \\
\hline $\mathrm{GMI}_{1.25}$ & 10.872 & 10.173 & 27.405 & 27.982 & 11.903 & 11.279 & 26.618 & 27.086 \\
\hline $\mathrm{GMI}_{1.00}$ & 9.998 & 9.232 & 28.133 & 28.825 & 10.438 & 9.709 & 27.758 & 28.387 \\
\hline $\mathrm{GMI}_{0.75}$ & 9.555 & 8.786 & 28.526 & 29.254 & 10.010 & 9.257 & 28.122 & 28.801 \\
\hline $\mathrm{GMI}_{0.50}$ & 9.370 & 8.568 & 28.696 & 29.473 & 9.548 & 8.740 & 28.533 & 29.300 \\
\hline
\end{tabular}

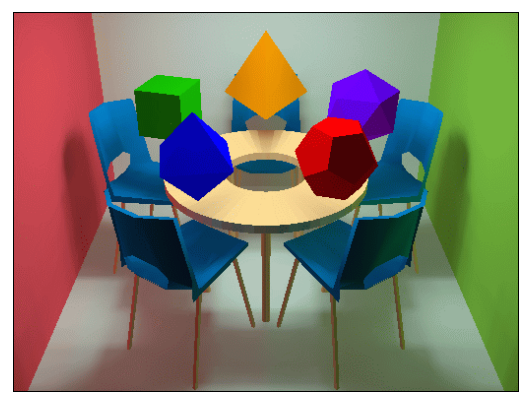

$(i)$

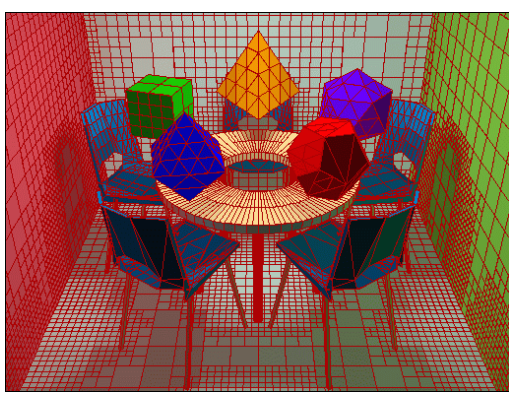

(ii)

Fig. 2. $\mathrm{GMI}_{0.50}$ oracle: (i) Gouraud shadow solution and (ii) mesh are shown

Table 2. The RMSE and PSNR measures of the $\mathrm{GMI}_{\alpha}$ oracle applied to the scene of Fig. 2, where the $\mathrm{GMI}_{0.5}$ result is shown. The oracle has been evaluated with 10 random lines between each two elements.

\begin{tabular}{|l||r|r||r|r|}
\hline oracle & RMSE $_{\mathrm{a}}$ & RMSE $_{\mathrm{p}}$ & PSNR $_{\mathrm{a}}$ & PSNR $_{\mathrm{p}}$ \\
\hline \hline $\mathrm{GMI}_{1.50}$ & 16.529 & 15.530 & 23.766 & 24.307 \\
\hline $\mathrm{GMI}_{1.25}$ & 15.199 & 14.145 & 24.494 & 25.119 \\
\hline $\mathrm{GMI}_{1.00}$ & 14.958 & 13.844 & 24.633 & 25.306 \\
\hline $\mathrm{GMI}_{0.75}$ & 14.802 & 13.683 & 24.724 & 25.407 \\
\hline $\mathrm{GMI}_{0.50}$ & 14.679 & 13.573 & 24.797 & 25.477 \\
\hline
\end{tabular}

In general, the improvement obtained with the $\mathrm{GMI}_{\alpha}$ oracle is significant. Moreover, its behaviour denotes a tendency to improve towards subextensive entropic indexes $(\alpha<1)$. To observe this tendency, another test scene is shown in Fig. 2 for an entropic index 0.5. Its corresponding RMSE and PSNR measures are presented in Table 2. The meshes are made up of 10,000 patches with 9,268,000 rays to distribute the power and we have kept 10 random lines to evaluate the oracle between elements. 


\section{Conclusions}

We have presented a new generalised-mutual-information-based oracle for hierarchical radiosity, calculated from the difference between the continuous and discrete generalised mutual information between two elements of the adaptive mesh. This measure expresses the loss of information transfer between two patches due to the discretisation. The objective of the new oracle is to reduce the loss of information, obtaining an optimum mesh. The results achieved improve on the classic methods significantly, being better even than the version based on the Shannon mutual information. In all the tests performed, the best behaviour is obtained with subextensive indexes.

Acknowledgments. This report has been funded in part with grant numbers: IST-2-004363 of the European Community - Commission of the European Communities, and TIN2004-07451-C03-01 and HH2004-001 of the Ministry of Education and Science (Spanish Government).

\section{References}

1. Goral, C.M., Torrance, K.E., Greenberg, D.P., Battaile, B.: Modelling the interaction of light between diffuse surfaces. Computer Graphics (Proceedings of SIGGRAPH '84) 18(3) (July 1984) 213-222

2. Hanrahan, P., Salzman, D., Aupperle, L.: A rapid hierarchical radiosity algorithm. Computer Graphics (Proceedings of SIGGRAPH '91) 25(4) (July 1991) 197-206

3. Feixas, M., del Acebo, E., Bekaert, P., Sbert, M.: An information theory framework for the analysis of scene complexity. Computer Graphics Forum (Proceedings of Eurographics '99) 18(3) (September 1999) 95-106

4. Feixas, M.: An Information-Theory Framework for the Study of the Complexity of Visibility and Radiosity in a Scene. PhD thesis, Universitat Politècnica de Catalunya, Barcelona, Spain (Desember 2002)

5. Taneja, I.J.: Bivariate measures of type $\alpha$ and their applications. Tamkang Journal of Mathematics 19(3) (1988) 63-74

6. Havrda, J., Charvát, F.: Quantication method of classication processes. Concept of structural $\alpha$-entropy. Kybernetika (1967) 30-35

7. Gortler, S.J., Schröder, P., Cohen, M.F., Hanrahan, P.: Wavelet radiosity. In Kajiya, J.T., ed.: Computer Graphics (Proceedings of SIGGRAPH '93). Volume 27 of Annual Conference Series. (August 1993) 221-230

8. Feixas, M., Rigau, J., Bekaert, P., Sbert, M.: Information-theoretic oracle based on kernel smoothness for hierarchical radiosity. In: Short Presentations (Eurographics '02). (September 2002) 325-333

9. Rigau, J., Feixas, M., Sbert, M.: Information-theory-based oracles for hierarchical radiosity. In Kumar, V., Gavrilova, M.L., Tan, C., L'Ecuyer, P., eds.: Computational Science and Its Applications - ICCSA 2003. Number 2669-3 in Lecture Notes in Computer Science. Springer-Verlag (May 2003) 275-284

10. Bekaert, P.: Hierarchical and Stochastic Algorithms for Radiosity. PhD thesis, Katholieke Universiteit Leuven, Leuven, Belgium (December 1999) 
11. Tsallis, C.: Possible generalization of Boltzmann-Gibbs statistics. Journal of Statistical Physics 52(1/2) (1988) 479-487

12. Tsallis, C.: Generalized entropy-based criterion for consistent testing. Physical Review E 58 (1998) 1442-1445

13. Taneja, I.J.: On generalized information measures and their applications. In: Advances in Electronics and Electron Physics. Volume 76. Academic Press Ltd. (1989) 327-413

14. Rigau, J.: Information-Theoretic Refinement Criteria for Image Synthesis. PhD thesis, Universitat Politècnica de Catalunya, Barcelona, Spain (November 2006) 